\title{
On Chinese and English Metaphors of Taste Based on Conceptual Metaphor Theory: A Case Study of Taste Word Translation in Fortress Besieged
}

\author{
Lingli Li \\ School of Foreign Language and Literature, Shandong University, Ji'nan, China
}

\section{Email address:}

carol0713@163.com

\section{To cite this article:}

Lingli Li. On Chinese and English Metaphors of Taste Based on Conceptual Metaphor Theory: A Case Study of Taste Word Translation in Fortress Besieged. International Journal of Applied Linguistics and Translation. Vol. 7, No. 2, 2021, pp. 69-75.

doi: $10.11648 /$ j.ijalt.20210702.16

Received: May 9, 2021; Accepted: May 25, 2021; Published: June 21, 2021

\begin{abstract}
Metaphor is very common in literature. Conceptual metaphor theory (CMT) provides a brand-new way to study metaphor from cognitive perspective. This paper applies corpus analysis toolkit Antconc to analyze Chinese and English metaphors of taste based on the corpus of Fortress Besieged written by Ch'ien Chung-shu and its translation by Jeanne Kelly and Nathan K. Mao. It adopts quantitative and qualitative methods to analyze the taste words in Fortress Besieged and their translation methods, discusses the similarities and differences between the metaphorical meaning of Chinese and English taste words, hoping to help with future translation practice. Through data collection and analysis, “酸”(sour), “甘/甜”(sweet), “苦”(bitter) and “辣”(spicy/hot) turn out to be the most commonly used metaphors of taste to elucidate the characters' personalities and experiences in the novel. However, the metaphors of taste in the original text of Fortress Besieged are more commonly used than that in its translation and the metaphorical meaning of taste words is richer in Chinese. Although, there are some similarities between Chinese and English metaphors of taste, there are much more differences. From the perspective of CMT, the metaphors of “酸”, “甜/甘”, “苦” and “辣” belong to the structural metaphors and are mapped from taste domain to the domains of physiology, emotion, life, sleep, visual sense, degree, personality and conduct, while the metaphors of "sour", "sweet", "bitter" and "spicy/hot" are hardly mapped to physiology, degree and conduct domain. To deal with this, translators can use free translation and amplification translation methods, omitting, substituting the original vehicles in source text or adding explanations to achieve functional equivalence during translation.
\end{abstract}

Keywords: Conceptual Metaphor Theory, Metaphor Translation, Taste Words, Fortress Besieged

\section{Introduction}

Metaphor is a universal linguistic phenomenon and an important way for human beings to recognize the world. Before 1980s, the study of metaphor was confined to figure of speech. Under the influence of cognitive linguistics, metaphor is regarded as the manifestation of people's cognitive activities and researchers increasingly shift their attention to cognitive perspective. With in-depth study of cognitive metaphor, metaphor and metaphor translation researches based CMT are gradually given more and more attention nowadays.

Taste word is a kind of vocabulary that describes how people respond to the stimulus of taste buds. Both Chinese and English have systematic vocabulary of taste words. Based on the taste buds of the tongue, people generally feel sour, sweet, bitter, spicy, salty, astringent, etc. Although taste is basically the same, how people respond to the taste, in other words, the usage of synaesthesia, is not exactly the same, which is evident among different nationalities. Also, synaesthesia can not be simply understood as a lexical feature but should be explored from the cognitive metaphor system [1]. Considering the polysemy of tastes words which are frequently used in literary works, it is of significance to study the metaphors of taste from a cognitive perspective.

This paper analyzes Chinese and English metaphors of taste based on the corpus of Fortress Besieged written by Ch'ien Chung-shu and its translation by Jeanne Kelly and 
Nathan K. Mao. It adopts quantitative and qualitative methods to analyze the taste words “酸”(sour), “甘/ 甜”(sweet), “苦”(bitter), “辣”(spicy/hot) and their translation. Through inductive analysis of real translation corpus, it summarizes and demonstrates the translation rules of taste word metaphors, making up for the subjectivity and one-sidedness of previous researches, aiming to explore the similarities and differences between the metaphorical meaning of Chinese and English taste words and provide reference for future metaphor translation practice.

\section{Literature Review}

\subsection{Previous Studies on Metaphor}

In the past, metaphor studies were restricted to rhetoric. Chinese rhetoric generally considers metaphor to be a typical rhetorical device. It is a kind of conventional linguistic skill that processes the linguistic form to enhance the power of language expression. In Western rhetoric study, figures of speech were ever divided into syntagmatic figures of speech and paradigmatic figures of speech. In this classification, metaphor belongs to the paradigmatic one because it is a kind of word replacement.

The first person to classify metaphorical research theories was British-America philosopher Max Black. He divides metaphor theories into Comparison Theory, Substitution Theory and Interaction Theory, which are regarded as traditional metaphor studies. In 1936, English rhetorician Ivor A. Richard put forward Interaction Theory for the first time in The Philosophy of Rhetoric, becoming the third most influential metaphor theory after being improved by Black. The study of metaphor in the second half of the 20th century achieved unprecedented development under the background of cognitive science. From the perspective of cognition, human's conceptual systems, activities and languages are all constructed through metaphors. Based on Interaction Theory, Lakoff and Johnson proposed CMT which regards the forming of metaphor as a result of projection between two conceptual domains. They consider that the nature of metaphor is to understand and experience one kind of thing in accordance with another [2].

After the 1980s, more precisely after Liu Ningsheng translated parts of Metaphors We Live By, China's metaphorical research has actually made a major breakthrough.

Until now, domestic scholars have introduced, explained and constructively criticized the metaphoric theories such as MTC, Conceptual Blending Theory, etc. Besides, they also have made use of their linguistic and disciplinary advantages to investigate the specific expressions of metaphors in various fields and aspects, make cross-language comparisons, sort out similarities and differences, and understand the profound rationale behind these phenomena, such as the integration of metaphor study with cognitive neurology, pragmatics, and second language acquisition [3].

\subsection{Previous Studies on Metaphor Translation}

Metaphor is a difficult problem in translation practice and translation studies. The two questions that people often discuss are whether metaphors can be translated and metaphor translation methods.

Peter Newmark divides metaphor into six classes, that is, dead, cliché, stock, recent, original, and adapted metaphors [4]. In Approaches to Translation, Peter Newmark put up with seven types of translation methods of stock metaphor, also called standard metaphor. The main ideas of these translation methods are substitution, deletion, translating metaphor into simile, literal translation with explanation as well as literal translation, free translation and using the two translation methods simultaneously.

Raymond van den Broeck realized that culture was the source of potential contradictions in metaphor translation of all fields, and proposed different metaphor translation methods from Newmark, including translation 'sensu stricto', substitution and paraphrase [5].

In addition, some cognitive linguists, such as Zoltán Kövecses, proposed more comprehensive metaphor translation methods according to the mapping conditions of metaphors.

In China, Guo Yingzhen compares Chinese and English metaphors from the perspective of pragmatics and culture. He proposes using literal and free translation at the same time in the translation of English and Chinese metaphors. Similarly, some scholars like Xiao Jiayan, Zhang Peiji, and Guo Zhuzhang believe that metaphor translation activities are potentially influenced by such factors as social, national culture, and literary traditions that metaphors rely on, put forward metaphor translation methods in respectively.

\subsection{Previous Studies on Metaphor Translation in Fortress Besieged}

Some studies have been made on metaphor translation in Fortress Besieged. According to the statistics of CNKI and Web of Science from 2015 to 2020, these studies are mainly qualitative studies from the traditional or linguistic approach. The research objects range from lexical metaphors to sentential metaphors, from conventional metaphors to novel metaphors.

From the traditional approach, considering metaphor as a figure of speech, Guo Xiaohua probed into the lexical and sentential metaphor translation based on eco-translatology [6]. Zhang Yayun studied the metaphor translation based on the seven translation methods put forward by Peter Newmark, and furthermore analyzed the reasons of translators' rendition deviation [7]. However, the given examples are not fully supportive. Cheng Tongtong and Wang Jin'an studied the applied ethic models during translation [8].

From the linguistic approach, Cai Xiaoyan studied the translation of metaphors in Fortress Besieged in terms of pragmatic enrichment [9]. Based on cognitive perspective, some studies revolve around C-E metaphor translation principles and methods in Fortress Besieged based on such 
translation theories as Relevance Theory, functional equivalence [10-12]. Wang Yinping explored its metaphor translations of nouns, idioms and ironic expressions [13], and Tian Dan and Fan Jingjing studied the metaphor translation related to human in Fortress Besieged [14].

\section{Theoretical Framework}

\subsection{Conceptual Metaphor Theory}

Metaphor translation, in terms of its operating process, can be understood as the integration of conceptions and languages between the source language and the target language. CMT was put forward by Lakoff and Johnson who implied that the essence of conceptual metaphor is experiencing and understanding one kind of thing in terms of another.

As for the working mechanism of CMT, it involves a one-way cross-domain mapping of two cognitive domains from source domain to target domain. The two domains must be similar and share common attributes. And the mapping between the two domains is based on the principle of consistency, which means that the image schema structure in the source domain is projected to the target domain in a way consistent with the internal structure of the target domain. For example, in the metaphor "life is a journey", life is the source domain, and journey is the target domain. Both of the two domains enjoy a structure of distance. The elements in the source domain contain people, birth, growth and death, while the elements in the target domain include traveler, starting point, experiences and destination.

Besides, Lakoff and Johnson divided conceptual metaphors into three categories, that is, structural metaphor, orientational metaphor and ontological metaphor. Structural metaphor interprets one kind of unfamiliar and abstract concept by using a familiar and concrete concept or structure. For example, in the sentence "you're wasting my time", time as a vehicle is compared with money to make the sentence more vivid. Orientational metaphor refers to the use of spatial concepts, such as up and down, inside and outside, front and back, near and far, center and edge, to understand another conceptual system. For instance, I'm feeling up. "Up" is used to express the happy feeling. Ontological metaphor mainly refers to the transformation of non-natural objects, such as abstract emotions or concepts, with natural objects which have spatial boundaries and volumes, so that we can describe them accordingly in terms of materiality, such as reference, categorization, classification and quantification. For example, in the sentence "if there's much more inflation, we'll never survive", the abstract concept "inflation" is quantified through the use of "much more".

Furthermore, two kinds of ontological metaphors, container metaphor and anthropomorphic metaphor are frequently used. Container metaphor refers to considering non-container or fuzzy concepts, such as vision, events, behaviors, activities, etc., as containers. Anthropomorphic metaphor is to give the object some characteristics of human beings so that the tenor can be understood through human motivation, characteristics, activities and so on.

\subsection{Functional Equivalence Theory}

The Functional Equivalence Theory proposed by Eugene A. Nida, an American translator, refers to the equivalence in meaning and function between the translated text and the original text, rather than in form and structure. The information conveyed by the translation should not only reflect the lexical meaning of the original text, but also contain its deep cultural connotations [15]. And its core is that the response of the target text readers is basically the same as that of the original text readers [16]. This theory requires the translator to fully understand the meaning of the source text, and then to convey it in the linguistic form that the target language readers are accustomed to, and at the same time, the target text should retain the style and cultural connotation of the source text to the maximum extent, so as to achieve the same response between the target language readers and the source language readers.

Based on Functional Equivalence Theory, three kinds of metaphor translation methods can be applied, that is, literal translation, free translation and amplification. To remain the same cultural connotation as the source text, translators can use literal translation to retain the original vehicle of the metaphor. If the original vehicle makes the target readers difficult to understand the translation, it is better to adopt free translation method and omit or substitute the original vehicle to keep the translation natural and fluent. In order to both retain the original cultural connotation and keep the target text natural and fluent, the translator can use amplification translation method, by which the translator retains the original vehicle and adds some explanations.

\section{Methodology}

\subsection{Research Questions}

This paper analyzes the metaphors of taste in Fortress Besieged from the perspective of CMT as well as Jeanne Kelly and Nathan K. Mao's translation methods based on Functional Equivalence Theory in order to tackle the following research questions:

1. What kinds of taste words are commonly used in Fortress Besieged?

2. How these metaphors of taste words are used according to CMT?

3. What are the differences and similarities between Chinese and English metaphors of taste?

\subsection{Data Collection and Analysis}

This paper uses corpus analysis toolkit Antconc which was developed by Laurence Anthony, a professor of Waseda University, to identify and categorize the data. First of all, ROST CM6 software was used for word segmentation of the original text of Fortress Besieged. After that, the processed corpus was saved as TXT file and UTF-8 format was used for encoding. Then, the processed source text was uploaded onto 
Antconc, and tastes words, such as “酸”, “甜/甘”, “苦”, “辣”, “咸”, etc., were used as search terms to find out how many times they had appeared. It turns out that “酸”, “甜/甘”, “苦”, “辣” are most frequently used in the source text. Then, the translated text was uploaded onto Antconc to search equivalent terms "sour", "sweet", "bitter" and "spicy/hot". The number of “酸”(sour), “甘/甜”(sweet), “苦”(bitter) and “辣”(spicy/hot) is counted as the following:

Table 1. Frequency of “酸”(sour), “甘/甜”(sweet), “苦”(bitter) and “辣”(spicy/hot).

\begin{tabular}{lllll}
\hline & 酸(Sour) & 甘/甜(Sweet) & 苦(Bitter) \\
\hline Source text & 23 & $11 / 19$ & 66 & 辣(Spicy/Hot) \\
Target text & 6 & 47 & 8 & 6 \\
\hline
\end{tabular}

Through quantitative analysis, it is found that the number of “酸”, “甜/甘”, “苦” and “辣” in the original text are 23, 11/19, 66 and 6 respectively, while the number of "sour", "sweet", "bitter" and "spicy/hot" in the translation are 9, 47, 8 and 2/35 respectively. Although the word "hot" appears frequently, there is only one case corresponding to "spicy", and most of other items "hot" means having a high temperature. It can be seen that the frequency of taste words in Chinese is very different from that in English, and thus the metaphorical meaning of taste words in Chinese and English is very different.

Besides, context which contains linguistic context, context of situation and context of culture is the basis of identifying and understanding metaphors. Metaphor identification procedure (MIP), put forward by Pragglejaz Group, was applied to detect metaphors related to “酸”, “甜/甘”, “苦” and “辣”. The main contents of MIP are as follows. First, read through the targeted text to form an overall understanding of textual meaning. Second, determine lexical units of the text and the contextual meaning of each lexical unit, that is, how the lexical unit refers to entities, relations or characteristics in specific context, especially considering the linguistic context and context of situation. For each lexical unit, first determine whether it has a more basic meaning in another context, and then compare the basic meaning and contextual meaning of the lexical unit to determine whether it is possible to understand the contextual meaning through the comparison between them. If possible, this lexical unit is labeled as a metaphor. If the contextual meaning cannot be understood after comparison, the lexical unit is not regarded as a metaphor.

After the identification and analysis of metaphor in the original text of Fortress Besieged, it is found that in most cases the taste words “酸”, “甜/甘”, “苦” and “辣” are not the original meaning of their taste domain but the metaphorical meaning which is projected to other domains. The following tables show the mapping domains, numbers and examples of the metaphors of “酸”, “甜/甘”, “苦” and “辣”.

Table 2. Mapping of “酸”.

\begin{tabular}{lll}
\hline Target domain & Numbers & Examples \\
\hline Physiology & 11 & “腰肢酸痛”, “眼酸”, “胳膊酸半天” \\
Emotion & 8 & “心里酸溜溜的”, “心酸啜泣”, “做酸泼醋”, \\
\hline
\end{tabular}

Table 3. Mapping of “甜/甘”.

\begin{tabular}{|c|c|c|}
\hline Target domain & Numbers & Examples \\
\hline life & 3 & “苦尽甘来”, “甜头吃完了”, “路上就甜了” \\
\hline sleep & 3 & “这一晚的睡好甜”, “黑甜乡”, “甜睡” \\
\hline Visual sense & 5 & “黑甜”, “笑起来甜甜的” \\
\hline Emotion & 12 & “温柔甜蜜”, “甜蜜地执拗”, “心有不甘” \\
\hline
\end{tabular}

Table 4. Mapping of “苦”.

\begin{tabular}{lll}
\hline Target domain & Numbers & Examples \\
\hline Emotion & 19 & “何苦”, “心融化成苦水”, “心里的痛苦” \\
Visual sense & 9 & “满脸苦笑”, , “苦尽来”, “苦经” \\
Life & 14 & “过去吃不来苦”, “苦”路上辛苦”, “苦事”, “年轻人应当苦一点” \\
Physiology & 18 & “苦劝” \\
Degree & 4 & \\
\hline
\end{tabular}

Table 5. Mapping of “辣”.

\begin{tabular}{lll}
\hline Target domain & Numbers & Examples \\
\hline Personality & 2 & “肥㖑辛辣的引力”, “泼辣” \\
Conduct & 2 & “下辣手”, “手段老辣” \\
\hline
\end{tabular}

Based on the above tables, it is easy to analyze the metaphorical meaning of “酸”, “甜/甘”, “苦” and “辣” in Fortress Besieged. “酸” is mapped to the domains of physiology and emotion. “甜/甘” is mapped to the domains of life, sleep, visual sense and emotion. “苦” is mapped to the domains of emotion, visual sense, life, physiology and degree. And “辣” is mapped to the domains of personality and conduct. All of these metaphors are structural metaphors in terms of the three categories of conceptual metaphor. However, English taste words do not necessarily have the same projection in 
these target domains, so translators need to adopt different methods in the process of dealing with the translation of these taste words.

\section{Analysis of Metaphors of Taste and Their Translations in Fortress Besieged}

This part selects some examples related to metaphors of “酸”, “甜/甘”, “苦” and “辣” in Fortress Besieged, analyzes their translation based on the translation methods of literal translation, free translation and amplification, and discusses the similarities and differences between Chinese and English metaphors of taste.

\subsection{Literal Translation}

Example 1

Source Text: 鸿渐吻她, 把这句话有效地截断, 然后说: “你今天真是颗酸葡萄” [17]。

Target Text: He kissed her, effectively cutting off her sentence, and said, "You sure are a sour grape today" [18].

Sun Jou-chia minded Fang Hung-chien's previous romantic experiences very much and thought he did not like her. In this example, Fang Hung-chien considers Sun Jou-chia as a sour grape. The word "酸” in the taste domain is mapped onto the emotion domain and corresponds to "jealousy" in the target domain. However, the expression "sour grape" also exist in English to express that someone is pretending that they dislike something because they want it but cannot have it. This metaphorical meaning is similar in Chinese and in English. Thus, the translators adopt literal translation and keep the original vehicle "sour grape" to maintain the artistic feature of the source text.

Example 2

Source Text: 他想这一晚的睡好甜, 充实得梦都没做, 无怪睡口丩 “黑甜乡” [17]。

Target Text: He reminisced how sweet the night's sleep had been, too deep even for dreams. No wonder sleep was called the land of dark sweetness [18].

In example 2, Fang Hung-chien and Miss Pao had one-night stand. He described his dream that night to be sweet. The taste word “甜” is mapped onto the domain of sleep and corresponds to "smooth" or "satisfactory" in the target domain. As "sweet" also has such metaphorical meaning in English, the translators retain the vehicle "sweet" and translate “黑甜乡” into "the land of dark sweetness" literally.

\subsection{Free Translation}

\subsubsection{Omission of the Vehicle of Metaphor}

\section{Example 3}

Source Text: 鸿渐闹别扭要辞, 经不起辛相苦劝, 并且傍 晚高松年亲来回拜, 终于算有了面子, 还是去了 [17]。

Target Text: Hung-chien fussed and refused to go but was unable to withstand Hsin-mei's earnest entreaties, and when early that evening Kao Sung-nien came by to pay a call, he felt he had sufficiently recovered face and went after all [18].

In example 3, “苦劝” contains a metaphorical meaning. The taste word “苦” is mapped from the taste domain to the domain of degree to describe the earnestness of Hsin-mei's expostulation. However, the word "bitter" in English hardly express the degree and literal translation will cause confusion. Thus, the translators omit the vehicle and translates “苦劝” into “earnest entreaties” to keep the translation natural and fluent.

Example 4

Source Text: 你这委曲求全的气量真不痛快! 做领袖有 时也得下辣手 [17]。

Target Text: Your tendency to concede in order to accommodate everyone is really getting us nowhere! A leader sometimes has to be ruthless [18].

In this example, Hung-chien complained that Hsin-mei was too soft in the way he had given in to Mr. Li at every turn. According to the context, “辣” is metaphorically used. It is mapped from the taste domain to the conduct domain. “辣” correspond to the venomous way of doing things. However, neither spicy nor hot has the same metaphorical meaning as “辣” in Chinese. Thus, the translators adopt free translation and omit the vehicle “辣手”, translating “下辣手” into “be ruthless".

\subsubsection{Substitution of the Vehicle of Metaphor}

Example 5

Source Text: 柔嘉也倒在沙发里心酸啜泣。鸿渐看她哭 得可怜, 而不愿意可怜, 恨她转深 [17]。

Target Text: Jou-chia collapsed on the sofa sobbing bitterly. Hung-chien watched her crying piteously, but he would not pity her and only hated her all the more [18].

In this example, Hung-chien hit Jou-chia, so Jou-chia was inconsolable and desired to break up with Hung-chien. In the expression “心酸啜泣”, “酸” is mapped from the taste domain to the emotion domain, referring to sadness in the target domain. However, "sour" does not have such metaphorical meaning in English, while "bitter" can be metaphorically used to express somebody's upset feeling. So, the translators substitute "sour" with "bitter" and translate “心酸啜泣” into “sobbing bitterly”.

\subsection{Amplification}

Example 6

Source Text: 方鸿渐自觉本日运气转好, 苦尽甘来 [17]。 Target Text: On the other hand, Fang Hung-chien decided his luck that day had turned for the better, as in the expression "After the bitter comes the sweet" [18].

In this example, “苦尽甘来” is a Chinese four-character idiom, implying the happy days will come after suffering. The taste words “苦” and “甘” are mapped to the domain of life, corresponding to the hardship and happiness in life. Although there is not such expression in English, the target text readers can understand it from the context. Thus, the translators adopt amplification translation method, retaining the vehicle and adding "as in the expression" and quotation marks so as to imply the unique Chinese culture and achieve functional equivalence to the maximum during translation. 


\section{Example 7}

Source Text: 辛椐一肚皮的酒, 几乎全成酸醋 [17]。 Target Text: The wine in Hsin-mei's stomach turned to sour vinegar in his jealousy [18].

In Example 7, seeing that Miss Su dabbed a little of the Tiger Balm and applied it to Hung-chien's temples, Hsin-mei was jealous. Here, “醋酸” does not actually exist but conveys a metaphorical meaning. “酸” is mapped from the taste domain to the emotion domain and corresponds to jealousy in the target domain of emotion. In order to maintain the style of original text and make the context meaning easily understood by target text readers, the translators adopt amplification method, retaining the original vehicle "sour vinegar" and adding "in his jealousy".

\subsection{Discussion}

There are a large number of metaphors of taste in Chinese which we often unconsciously use in our daily life. Viewing from the collected data, it is easy to find that the taste words “酸” and “苦” in the source text of Fortress Besieged appear much more frequently than their equivalents in the target text, while the corresponding English taste words of “甘/甜” and “辣”, in other words, “sweet” and "spicy/hot” present themselves more often than that in the original text. In the original text, most of the taste words “酸”, “甜/甘”, “苦” and “辣” are metaphorically used, among which the metaphors of “酸”, “甘/甜” and “苦” account for a large proportion, with the word frequency of 19, 23 and 64 respectively. However, in the target text, "spicy/hot" refer to their literal meaning in most contexts.

Thus, based on all of the above analysis, it turns out to be that the metaphors of taste in the original text of Fortress Besieged is more commonly used than those in its translation and the metaphorical meaning of “酸”, “苦” and “辣” is richer in Chinese text, while the opposite is true for “甜/甘”. Although, there are some similarities between Chinese and English metaphors of taste, there are much more differences. For example, both "酸” and "sour" can be mapped onto the emotion domain. They can refer to jealousy in the target domain but "酸” can also refers to sadness in Chinese. And the word "sour" is hardly ever mapped onto the physiology domain in English. “苦” in Chinese also contains richer metaphorical meaning than "bitter". Apart from the emotion domain and domain of visual sense, such as “苦笑/smile bitterly”, “苦” also includes more connotations in the life, psychology and degree domains in Chinese.

The underlying reasons for the differences in metaphorical meaning of taste words can be analyzed as follows: Chinese and western cultures cherish different thinking modes. People in the east usually consider that man is an integral part of nature, emphasizing morality and ethics, while people in the west put more emphasis on cognition, analytical and rational thinking [19]. Thus, the taste words “酸”, “甜”, “辣” are mainly mapped to the domains related to human. This novel contains abundant details to illustrate the distinctive personalities, such as“做 酸泼醋”, and “泼辣” which are metaphorically presented in the original text but cannot be literally understood in English. Therefore, these vehicles are omitted or substituted in translation. In English, "sweet" can also be metaphorically used in character description, but it can also be mapped to many abstract concepts, such as "sweet joy of revenge”, to substitute the idiomatic Chinese “报了仇那样 的舒畅”.

Besides, Chinese culture is deeply influenced by Buddhism and Confucianism. "Bitterness" is a basic word of Buddhism. The whole Buddhist thought starts from suffering or "bitterness" and revolves around how to get rid of suffering to achieve happiness [20]. Also, there is traditional thought of "enduring hardship" in Confucianism and it has deeply influenced Chinese people for thousands of years. Thus, "bitter" is of importance in people's daily life and is given various extended meaning in Chinese.

What's more, the writing style of Fortress Besieged can also be found from the metaphorical use of taste words. Ch'ien Chung-shu is very good at using taste words to describe the main characters' personalities. For example, Miss Su's "sweet, cool ice cream manner," and "Dark Sweetness" Miss Pao. And the translators used literal translation methods according, faithfully conveying the writing style of the original text.

\section{Conclusion}

Due to the different cultures and thinking modes, although there are some similar meanings in Chinese and English metaphors of taste, there are also many differences among them. This paper combines the cognitive metaphor study with functional equivalence theory, adopts the methods of quantitative and qualitative analysis to explore the metaphors of taste words in Fortress Besieged as well as the similarities and differences between English and Chinese metaphors of taste words.

Through data collection and analysis, “酸” (sour), “甘/甜” (sweet), “苦” (bitter) and “辣” (spicy/hot) turn out to be the most commonly used metaphors of taste to express the characters' personalities and experiences in the novel, but most of these Chinese metaphors of taste share richer connotations than the equivalent English metaphors of taste. From the perspective of CMT, the metaphors of “酸”, “甜/甘”, “苦” and “辣” belong to the structural metaphors and are mapped from taste domain to the domains of physiology, emotion, life, sleep, visual sense, degree, personality and conduct, while the metaphors of "sour", "sweet", "bitter" and "spicy/hot" also are hardly mapped to physiology, degree and conduct domain. To deal with this, translators can use free translation and amplification translation methods, omitting, substituting the original vehicles in source text or adding explanations to achieve functional equivalence during translation.

However, because the corpus of this study is limited, the study of metaphorical meaning of “酸” (sour), “甘/甜” (sweet), “苦” (bitter) and “辣” (spicy/hot) may be not comprehensive. Further study can be conducted based on 
larger corpus to find more similarities and differences between English and Chinese metaphors of taste. What's more, this study only involves the analysis of metaphorical projection of basic taste words in English and Chinese, and the metaphorical projection of other taste words needs to be discussed and studied in the future.

\section{References}

[1] Wang S H, \& Xu J. (2002). Synaesthesia and Conceptual Metaphor. Foreign Language Research, (03), 91-94+112.

[2] Lakoff, G., \& Johnson, M. (1980). Metaphors We Live By. Chicago: University of Chicago Press.

[3] Sun Y. (2020). A Rediscussion on Contemporary Metaphorology in China (2014-2018): A Bibliometric Survey Based on Journals of Foreign Language Studies Indexed by CSSCI. Foreign Language Research, (03), 50-55.

[4] Newmark, P. (1988). A Textbook of Translation. London: Prentice Hall.

[5] Van den Broeck, R. (1981). The Limits of Translatability Exemplified by Metaphor Translation. Poetics Today, 2, 73-87.

[6] Guo X H. (2016). On the Translation of Metaphor from the Perspective of Eco-translatology---a Case Study on Fortress Besieged. Journal of Shaanxi Xueqian Normal University, (03), 67-69.

[7] Zhang Y Y. (2017). Analyses of translations of metaphor in Fortress Besieged from the aspect of Newmark's translation method. Journal of Yuzhang Normal University, (05), 30-32.

[8] Chen T T, \& Wang J A. (2019). Study on the Ethic Models in Metaphor Translation of Fortress Besieged. Overseas English, (21), 132-133.
[9] Cai X Y. (2015). Metaphor Translation in Fortress Besieged from the Perspective of Pragmatics. Journal of Hubei University of Science and Technology, (01), 88-90.

[10] Xu Y T. (2016). Validity of Metaphor Translation in Fortress Besieged in light of Relevance Theory. Advances in Social Science, Education and Humanities Research, 85, 327-331.

[11] Yu X C, \& Wang Q S. (2017). On the Translation Methodology of Fortress Besieged from the Perspective of Functional Equivalence. Journal of Jiangsu Ocean University (Humanities \& Social Sciences Edition), (03), 69-73.

[12] $\mathrm{Hu}$ X K. (2017). Study on Metaphor Translation Strategies---Taking Fortress Besieged as an Example. Language Planning, (17), 69-70.

[13] Wang Y P. (2016). Study on Characteristics and Translation of Metaphors in Fortress Besieged. Language Planning, (03), $51-52$

[14] Tian D, \& Fan J J. (2020). Study on Translation Strategies of Conceptual Metaphors Related to Human. Overseas English, (10), $40-41+56$.

[15] Nida, E. A. (1964). Toward A Science of Translating. Leiden: E. J. Brill.

[16] Nida, E. A., \& Taber, C. R. (2004). The Theory and Practice of Translation. Shanghai: Foreign Language Education Press.

[17] Ch'ien Chung-shu. (2012). Fortress Besieged. Beijing: People's Literature Publishing House.

[18] Ch'ien, Chung-shu. (2003). Fortress Besieged. Trans. Kelly, J, $\&$ Mao, N K, trans. Beijing: Foreign Language Teaching and Research Press.

[19] Lian S N. (2002). On Chinese and Western Thought Patterns. Foreign Languages and Their Thinking, (02), 40-46+63-64.

[20] Yang L W. (2017). On the Origin of Several Basic Buddhist Words in Māgadhābhāsā and Related Thinking. Religious Studies, (03), 104-109. 